\title{
Study on the Clinical Manifestations and Diagnostic Criteria of Premature Ovarian Failure
}

\author{
Lili Xuan, Xiaojun Liu* \\ China-Japan Union hospital of Jilin University, Changchun, Jilin, 130033 \\ *corresponding author
}

Keywords: clinical manifestations; diagnostic criteria; premature ovarian failure

\begin{abstract}
Premature ovarian failure (POF) refers to a disease characterized by amenorrhea, infertility, estrogen deficiency, and elevated gonadotropin levels in women before 40-year-old. This paper reports that the incidence of premature ovarian failure is $1 \%$ before 40 and 1 before 30 . The prevalence of POF in primary amenorrhea patients is $10 \%$ to $28 \%$, and the POF incidence in secondary amenorrhea is $4 \% \sim 18 \%$. This paper briefly discusses its clinical manifestations and diagnostic criteria.
\end{abstract}

\section{Introduction}

The cause of premature ovarian failure is extremely complex, including many factors such as physiological pathology, mental health, and social environment. In terms of the body itself, it also includes various factors such as systemic diseases and local lesions of the reproductive organs [3]. The common clinical causes are as follows.

In retrospective history, about half of the patients with premature ovarian failure had family history of early menopause among the immediate or adjoining relatives of their mothers, aunts, and aunts. Patients are often the same as their elders, who begin menstrual disorders around the age of 35 to 38 years, and gradually amenorrhea, vaginal discharge is also reduced, there is a series of hot sweating, upset anxiety, insomnia, joint pain and other menopausal syndrome The clinical manifestations. Studies [4] have shown that approximately $10 \%$ of POF patients with sisters or three generations of the grandparents and grandchildren can develop joint disease, all of which show karyotypic abnormalities or gene mutations. This shows that the cause of this group of patients with premature ovarian failure is closely related to family genetics.

The frequency of sexual life of patients with premature ovarian failure is generally lower than that of women of normal reproductive age. They are often single unmarried or divorced, or long-term couples living apart from each other, or although living together, but due to a variety of factors such as the two sides or one of the causes of sexual life Deficiency, long-term sexual function is in a depressed or non-use state, and the erotic desire gradually decreases over time, leading to premature ovarian failure.

About one-third of patients with premature ovarian failure are poor or manual workers. Due to low economic income, long-term hard work or physical labor, or persistent frugality, prolonged malnutrition, or unbalanced nutrition, decreased protein and creatinine levels, symptoms of premature ovarian failure often occur. Not only is the appearance of old age, but also sexual dysfunction, premature menstruation. Acute blood loss is often due to childbirth, induction of labor, abortion, or medical abortion, or uterine bleeding, or medical diseases such as perforation of gastrointestinal ulcers, etc., transient massive blood loss, but also failed to timely transfusion supplement, so that the human body ischemic hypoxia The genital organs, including the ovaries, are also in a condition of insufficient blood supply, and even the appearance of irreversible premature ovarian failure such as Sheehan's syndrome. Chronic blood loss is often caused by chronic gastritis enteritis, chronic wasting diseases caused by human body digestion and absorption disorders, or anal fissure, hemorrhoids, etc., leading to chronic anemia, can also cause ovarian feeding failure, hormone secretion and premature aging. 
Unhealthy living habits Some patients with premature ovarian failure due to long-term partial eclipse, picky eaters, excessive diet to lose weight, resulting in uneven nutrition, lack of long-term ovarian supply, resulting in decreased ovarian secretory hormone function; also some patients with smoking, drinking, drug abuse and other bad habits, toxic and harmful substances Into the blood circulation, direct damage to ovarian function [3]; or long-term Internet access, reversed yin and yang, endocrine disorders, ovulation disorders, menstrual sparse, ovarian polycystic changes gradually develop to follicular atresia, atrophy, and gradually decline in function and premature aging.

\section{Laboratory Inspection}

Determination of serum hormone levels showed that elevated FSH levels and decreased estrogen levels are the most important features and diagnostic criteria for POF patients. Generally, FSH> 40 $\mathrm{U} / \mathrm{L}$ and estradiol $<732 \mathrm{pmol} / \mathrm{L}$. The most sensitive of these is elevated serum FSH, which is an early indicator of POF. Occasionally, patients with POF have temporary recovery of ovarian function. Continuous measurement of serum sex hormones found that almost half of POF women exhibited intermittent ovarian function recovery, that is, serum estradiol levels were above 183 $\mathrm{pmol} / \mathrm{L}$, and even nearly $20 \%$ of women could Occurrence of intermittent ovulation means serum progesterone levels exceed $95 \mathrm{nmol} / \mathrm{L}$ [4]. The pathophysiology of this phenomenon is similar to the transition period of menopause. During this period, the remaining follicles in the ovary still have intermittent activities, leading to fluctuations and instability of sex hormone levels. Therefore, only one measurement showed that elevated levels of FSH could not conclude that the ovarian function was completely depleted, and sometimes repeated measurements were required. Continuous elevation of FSH suggests that POF may be. It should be noted that serum FSH levels do not necessarily reflect the number of primordial follicles in the ovary, and that elevated FSH is only indicative of the negative feedback of sinusoidal follicles during development that lacks estrogen and inhibin. Pelvic ultrasonography in most patients with POF showed shrinking ovaries and uterus and no follicles in the ovary. However, more than one third of POF patients with normal karyotype have pelvic ultrasound examination and may have follicles. It is reported that after 6 years of confirmed premature ovarian failure, ultrasound can still detect the presence of follicles in the ovary, but in most women, these follicles do not have normal function. There is also no correlation between follicular diameter and serum estradiol levels [5]. There are two explanations for this phenomenon. One may be that there are indeed residual follicles in the ovary, and the other may be the so-called ovarian insensitive syndrome, which means that there are follicles in the ovaries, but it is not sensitive to FSH reactions and thus the follicles cannot develop. . It may be related to the defect of FSH receptor in the ovary. The exact etiology is not yet clear. It is difficult to identify with POF clinically. Ovarian biopsy found that more primordial follicles can diagnose. Ultrasonography can also detect abnormalities in the anatomy of the reproductive tract, such as malformations and absences of the reproductive tract. The clinical significance of detecting anti-ovarian antibodies is not yet known. There is no correlation between anti-ovarian antibodies and the severity of oophoritis, and it does not predict whether and when ovarian dysfunction will occur. Detection of commercially available kits may have 1 / 3 normal women with anti-nuclear antibody positive. Studies have shown that POF may occur in steroid-positive patients with adrenal failure. Patients with suspected autoimmune diseases should be examined for autoantibodies, ESR, immunoglobulins, and rheumatoid factor. When clinically indicated, thyroid function (blood thyroid hormone, thyroid stimulating hormone), adrenal function (blood and urinary cortisol, blood electrolytes), parathyroid function (parathyroid hormone), and blood glucose parameters can be measured.

\section{Diagnosis of Premature Ovarian Failure}

The recognized diagnostic criteria for premature ovarian failure are the presence of amenorrhea at least 4 months prior to the age of 40, and 2 or more serum FSH $>40 \mathrm{U} / \mathrm{L}$ (over two inspection 
intervals more than 1 month), estradiol level $<732$ pmol / L[1, 3]. Medical history, physical examinations, and other laboratory tests can help diagnose the underlying cause.

Detailed patient history was collected, including age at menarche, menstruation before amenorrhea, amenorrhea, presence or absence of amenorrhea (mental stimulation, environmental toxicants, etc.), history of drug use, history of cancer chemotherapy, radiotherapy history, History of ovarian surgery, history of pelvic infection, history of tuberculosis, and pregnancy and birth history. Subjective symptoms such as hot flashes, excessive sweating, insomnia, irritability, impatience, vaginal dryness, dysuria, etc. Past and present epidemic mumps and AIDS virus infections because of rare ovarian failure secondary to infection. To understand whether patients and their families have past and current autoimmune diseases such as Addison's disease, thyroid disease, diabetes, SLE, rheumatoid arthritis, leukoplakia, Crohn's disease, and Sjogren's syndrome [3]. A small number of epidemiological studies have shown that there are family trends in premature ovarian failure. Recent studies have also shown that genetic mutations in gonadotropin receptors can lead to premature ovarian failure. Therefore, family history should be carefully scrutinized, including the menstruation of mothers, sisters, and second-degree relatives of women. Situation and fertility of male relatives.

When a full-body examination is performed, attention is paid to overall development, intelligence, and nutritional status. Mammary glands and pubic hair development are examined and graded according to the Tanner grading standard [3]. Pelvic examination to observe whether there is atrophic vaginitis caused by lack of estrogen. Patients with autoimmune POF (lymphocytic ovary inflammation) can sometimes find enlarged ovaries by pelvic examination [3]. The focus should be on checking for signs related to the above autoimmune diseases. In addition to the determination of serum sex hormone levels, when there are clinical indications, attention should also be paid to the examination of relevant diseases as appropriate, such as blood, urine routine analysis, ESR, antinuclear antibodies, immunoglobulins, and rheumatoid factor tests. The presence or absence of pituitary tumors can be identified by magnetic resonance examination and by the production of intact FSH, and subunits by thyroid releasing hormone stimulation. People with suspected low bone mass and osteoporosis should be tested for bone mineral density. Pelvic ultrasonography was performed to see if there was an anatomical abnormality and the presence of follicles. However, in patients with spontaneous POF with normal karyotypes, pelvic ultrasonography does not change the clinical diagnosis, because even if a follicle is found, it has not been confirmed that treatment can restore ovarian function [3].

Kidney filling is the most important and most fundamental treatment for premature ovarian failure. It is clinically applicable to patients with premature ovarian failure who have a family history of hereditary disease and who have acquired kidney deficiency and acquired dystrophy. The clinical treatment should be based on the different treatment methods of kidney yin deficiency and kidney yang deficiency. In kidney-building, we must pay particular attention to the yin and yang's mutual rooting and understanding of the theory that "Yin is not born and there is no solitary yang". Mastering "good yin and yang will seek yin for the yin, and good yang to seek yang in the yin "the rules. In the early days, nourishing kidney yin was mainly used, with adjuvant therapy to help the yang, mid-nourishing yin and yang to aggravate the yang, and late qi to replenish yang to benefit yin, so that "your yin helps and the life is endless, yin yang rises and the spring is inexhaustible". Patients with chronic ovarian failure caused by chronic digestive diseases often have less energy, loose stools, fatigue, lack of complexion, emaciation, and low serum hemoglobin and ferritin levels. Chinese medicine believes that the spleen and stomach are the foundation of the acquired nature, the source of qi and blood, the diseases of the digestive system, the long-term digestion and malabsorption, malnutrition, the ingestion of the Migu subtle can not be smoothly transformed into blood, and nourish the organs, and of course it can not be properly supported. Ovaries, while the ovarian hormone secretion function gradually decreased, the negative feedback of the ovary on the pituitary also gradually weakened, will inevitably affect the normal regulation of the female reproductive physiology axis, caused by the elderly is not closed. Invigorating the spleen and stomach not only promotes the digestion and absorption of the spleen and stomach, and Huashui Gu 
is a qi and blood, but also helps the blood to spread through the body's internal organs and veins, and nutrition the ovary.

\section{Conclusion}

Patients with premature ovarian failure due to increased stress in life and work, or unspoken interpersonal relationships, or mental factors, long-term emotional uncontrollable, heart and liver qi stagnation, will inevitably affect the female kidney - Tianhao - Chong Ren - cell axis function, or see Heart and kidney do not pay, water and fire incompetence, or see the liver and kidney Yin Jing losses, rushed to the uterine palace, resulting in premature closure of the month, the tunnel is unreasonable. To regulate the function of the reproductive physiology axis, we must pay attention to both the heart and the liver. Heart blood, liver and blood; When tonifying the kidneys and spleen, it is necessary to coordinate with the heart and liver, raise blood, peace of mind, relieving the liver, raising liver and yin. Liver qi is so comfortable and his mind is very good that he can guarantee the smooth flow of blood and urine, yin and yang, and the continuity of the two veins.

\section{References}

[1] Chai Shujuan, Li Shuang, Summer. Pathogenesis of ovarian premature aging and prospects [J]. Hubei Traditional Chinese Medicine, 2010, 32(7): 76.

[2] Oasis. The incidence of premature ovarian failure and the knowledge, attitude, and behavior of young women aged 30 to 40 years [J]. Journal of Guangdong Medical College, 2014, 32(4) :565-567.

[3] Lv Qun, He Dongjie. The cause of premature ovarian failure and treatment progress [J]. Modern Clinical Medicine, 2014, 40(3): 225-229.

[4] Gong Cuiping, Hao Tianyu. Advances in the etiology of premature ovarian failure [J]. Journal of Liaoning Medical College, 2010, 31(3): 284.

[5] Pu Jiming. Diagnosis and treatment of premature ovarian failure [J]. Shanxi Traditional Chinese Medicine, 2013, 29(3):59. 